\title{
Selecting relevant and changeable determinants and underlying beliefs of university students' adherence to COVID-19-guidelines and translating the findings into a small intervention
}

Tugce Varol ${ }^{\mathrm{a}}$, Rik Crutzen ${ }^{\mathrm{b}}$, Francine Schneider ${ }^{\mathrm{b}}$, Ilse Mesters ${ }^{\mathrm{c}}$, Robert A.C. Ruiter ${ }^{\mathrm{a}}$, Gerjo Kok $^{\mathrm{a}}$, Gill ten Hoor*a

a. Department of Work and Social Psychology, Maastricht University, the Netherlands

b. Department of Health Promotion, CAPHRI, Maastricht University, The Netherlands

c. Department of Epidemiology, CAPHRI, Maastricht University, The Netherlands

*Corresponding author: Gill ten Hoor, Dept. Work \& Social Psychology, Maastricht

University, P.O. Box 616, 6200 MD Maastricht, The Netherlands

gill.tenhoor@maastrichtuniversity.nl 


\begin{abstract}
Background: When reopening universities in times of COVID-19, students still have to adhere to COVID-19 behavioral guidelines. We explored what behavioral determinants (and underlying beliefs) related to the adherence to guidelines are both relevant and changeable, as input for future interventions.
\end{abstract}

Methods: A cross-sectional online survey was conducted (Oct-Nov 2020), identifying behavioral determinants (and underlying beliefs) of university students' adherence to COVID19-guidelines, including keeping 1.5m distance, getting tested, and isolating $(N=255)$.

Results: Attitude, perceived norm, self-efficacy, and several beliefs (e.g., risk perception beliefs 'I am not afraid because I am young' $[r=-.33 ; p<.001]$; attitudinal beliefs, e.g., 'I feel responsible for telling people to adhere to guidelines' $[r=.37 ; p<.001]$; self-efficacy beliefs, e.g., 'COVID-19-prevention guidelines are difficult to adhere to' $[r=-.30 ; p<.001])$ were associated with intention to adhere to guidelines, and for those beliefs there was room for improvement, making them suitable as possible intervention targets.

Conclusions: Students mostly adhere to COVID-19 guidelines, but there is room for improvement. Interventions need to enhance students' adherence behavior by targeting the most relevant determinants as identified in this study. Based on these findings, a small intervention was introduced targeting the determinants of students' adherence to guidelines.

Keywords: COVID-19, university students, adherence, determinants, guidelines, intervention 


\section{Introduction}

Since the first identification of the coronavirus disease 2019 (COVID-19), almost all countries around the world took preventive measures. Person-to-person transmission of the virus was established as the source of infection (Adhikari et al., 2020; Rothan \& Byrareddy, 2020; Shereen et al., 2020), and therefore behavioral measures such as social distancing, quarantining and wearing facemasks were taken (Fauci et al., 2020; Van Bavel et al., 2020). Another measure was the closure of schools (ranging from primary schools to universities) to further slow the spread of the virus but with negative consequences for students' psychosocial wellbeing and educational development (Auger et al., 2020; Head et al., 2020; Petretto et al., 2020; Ziauddeen et al., 2020). Moreover, even though the vaccination has started, authorities continue to suggest people to follow the preventive measures as long as the vaccination rate has not reached a critical threshold for group immunity (WHO, 2020). Therefore, when reopening universities, university administrators need to develop and implement theory- and evidence-based interventions to enable students' safety within university facilities.

For intervention development, it is important to examine an individuals' relevant behaviors including the determinants and underlying beliefs of those behaviors (Bartholomew Eldredge et al., 2016; Fernandez et al., 2019; Peters, 2014). In this specific case, the focus is on students' behaviors and determinants. The role of the relevant stakeholders was already realized by the facilitation of preventive behaviors such as providing facilities for disinfecting hands, arrows for walking directions, instructions for taking a test, et cetera. In the present study, we answer the question why students perform specific risk behaviors and what motivates them to replace these behaviors with more safe behaviors. The identified belief structures, in turn, will serve as the target points for future interventions (Kok, 2014). 


\section{Theories about behavioral determinants and their underlying beliefs}

Theories behind this study included the Reasoned Action Approach (Fishbein \& Ajzen, 2010; Theory of Planned Behavior, Ajzen, 2011), which postulates that intention is the most proximal determinant of behavior. Intention is influenced by three other determinants with underlying beliefs: (a) attitude, people's evaluation of consequences and experiences when performing the behavior; (b) perceived norm, people's beliefs that important others would (dis)approve of their performing the behavior (injunctive norm) and their beliefs that others like themselves do (or do not) perform the behavior (descriptive norm); (c) perceived behavioral control (comparable to: self-efficacy), people's beliefs about the degree to which they are capable of, or have control over, carrying out the behavior. Protection Motivation Theory (Maddux \& Rogers, 1983) states that people respond to a threat based on its (a) perceived severity and (b) perceived susceptibility, (c) the availability of an adequate coping response, and (d) their self-efficacy to perform the behavior. Next to theories about reasoned behavior, theories on automatic behaviors and habits, which are context-dependent automatic behaviors (Wood \& Rünger, 2016), can additionally explain behavior, for example why it is difficult to keep distance from close friends with whom you normally might also be physically close with .

\section{Selecting the most relevant determinants by employing the CIBER approach}

After mapping the determinants/beliefs behind students' adherence behavior, the next step to create an intervention is to select the most relevant targets to intervene upon. To develop our intervention, we used the Confidence Interval-Based Estimation of Relevance (CIBER, [Peters and Crutzen, 2018]) approach, which enable intervention developers to select relevant determinants/beliefs based on 1) association between the behavior/intention and other determinants/beliefs and 2) room for improvement of each determinant/belief based on its univariate distribution. Although one determinant/belief might have a high correlation with 
intention/behavior, it still might not be a good target to incorporate in the intervention due to the less to no room for improvement. By room for improvement, we mean that, for instance, if people already show a high self-efficacy to adhere to the rules, this determinant cannot be substantially improved.

\section{The current study}

In this study, we aim to identify the relevant and changeable determinants and underlying beliefs of students' adherence to COVID-19-guidelines, thereby quantifying the findings of our earlier qualitative study (Varol et al., 2021). We use insights into the relevance of these determinants/beliefs in the intervention to be developed. Based on our qualitative findings, the behaviors that we focus on in this study are 1) adherence to general COVID-19 guidelines, and the two most important specific guidelines: 2) keeping at least $1.5 \mathrm{~m}$ distance, and 3) staying at home and getting tested when having symptoms. Although wearing a facemask inside facilities is also identified as an important measure, this was introduced as part of the university guidelines after the start of this study. Although we anticipated this by adding some belief-questions in our questionnaire, we did not measure intention or behavior, and therefore those outcomes are not reported here. Also washing/disinfecting hands is identified as important behavior, but compliance was already high, and therefore seen as having a lower need to change.

\section{Methods}

Strengthening the reporting of observational studies in epidemiology (STROBE) guidelines providing guidance to the researchers to report their studies were followed to report this observational study (Von Elm et al., 2007). In order to maximize scrutiny, foster accurate replication, and facilitate future data syntheses (e.g., meta-analyses) (Peters et al., 2012), study 
materials (e.g., questionnaire), as well as non-identifiable data, are available at the Open Science Framework: https://osf.io/43wpa/

\section{Participants and recruitment}

Participants were university students who had the intention to visit the Maastricht University buildings "within the next two months", as all questions were focused on that period (students filled out the online questionnaire between 26 October - 9 November 2020). They were recruited through Flycatcher (2021) (a certified online panel and operator of the existing representative student panel of Maastricht University (UM) - see also https://www.flycatcher.eu/en/Home/OverOns). This panel represented students from all Maastricht University faculties and programs. All students who are a member of the UM student panel were invited to participate in the online survey. As compensation for participating in a questionnaire through this panel, students receive a small incentive each time they participate in research (10 euro for 900 points and this survey was 150 points). This study was approved by the Ethics Review Committee Psychology \& Neuroscience, Maastricht University, ref. 188_10_02_2018_S59. All participants consented to participate in the study.

\section{Design and procedures}

Data collection period was between October 26 and November 9, 2020. In this period, the Dutch government installed an "intelligent lockdown" during which higher education institutions had the option to offer hybrid education in which students could choose between attending classes on-site within the university buildings (with a limited number of students being allowed in a time slot) or online. Students were invited to the study via e-mail, which included a hyperlink. When clicking to this link, they were directed to the survey which starts with the information about the questionnaire as well as a question whether they consent to participate in this study. Students who indicated that they do not want to consent were 
directed to the end of the survey. Students who consented received the questionnaire in blockrandom order in which the four categories were randomized over four orders (using a Latinsquare design, i.e. (1) ABCD; (2) DCBA; (3) BDAC; (4) CADB whereby A = general UM COVID-19-guidelines; $\mathrm{B}=$ keeping distance $\mathrm{C}=$ testing and isolating, and $\mathrm{D}=$ demographics/additional information - see also Measurements). Note that $\mathrm{A}$ is about behaviours specific to the university setting and $\mathrm{B}$ and $\mathrm{C}$ are more overarching because also applicable outside the university setting. No questions could be skipped, but participants were free to stop at any time. The language used in the questionnaire was English.

\section{Measurements}

In our earlier qualitative study (Varol et al., 2021), we gathered information on students' determinants and underlying beliefs regarding (non)adherence to the university's COVID-19-guidelines. Based on these findings and theories of reasoned and automatic behavior, we formulated our survey questionnaire. The items consisted of questions regarding 1) adherence to general COVID-19-guidelines of the university (keeping $1.5 \mathrm{~m}$ distance, disinfecting hands, refraining handshake, avoiding crowds and getting tested/isolating), 2) keeping $1.5 \mathrm{~m}$ distance, and 3) getting tested/isolating when having symptoms, and 4) demographics (i.e., gender, age, study-year, and faculty). The major reason that we measured demographics was to see if the sample would be comparable to the whole student panel (as we used the student panel for data collection). Lastly, some questions on education (hybrid vs. on-site and online vs. hybrid), social and mental health, and physical activity in times of COVID-19 were included, but these are not reported here as they are beyond the scope of this paper (see Supplementary File 1 for the complete questionnaire; see Supplementary File 2 for results). Determinants were measured based on the theories mentioned earlier and the content of the underlying belief items were created based on the information elicitated in the interviews with students. 


\section{Adherence to COVID-19-guidelines of the university}

After introducing the general COVID-19 prevention guidelines in university buildings, students' self-reported adherence to COVID-19-guidelines of the university (9 items) and intention to adhere to guidelines for the next two months (6 items) were measured. Those items related to 1) keeping $1.5 \mathrm{~m}$ distance from other people, 2) disinfecting hands upon entering the university building, 3) refrain from shaking hands, 4) avoiding crowds or situations where $1.5 \mathrm{~m}$ distance was not possible, 5) staying at home, and getting tested. To calculate a general adherence intention, we combined those intentions $(\Omega=.74)$ to one general adherence intention score. Please note that the university rule to wear a facemask was installed after we conducted this questionnaire, and therefore this intention was not included in this composite score. Additional to adherence behavior and intention, attitude (2 items; bad-good; unpleasant - pleasant), perceived norm (2 items; e.g., "Most people like me always adhere to the general university COVID-19 prevention guidelines"), self-efficacy (2 items; e.g., "I am confident that if I want to, I can adhere to the general university COVID-19 prevention guidelines"), risk perception beliefs (4 items, e.g., "I am not afraid of contracting COVID-19 because I am young”), attitudinal beliefs (7 items; e.g., “The general university COVID-19 prevention guidelines are irrelevant for our generation”), perceived norm belief (1 item, i.e., "My teachers/tutors at UM care about the general UM COVID-19 prevention guidelines"), and self-efficacy beliefs (2 items; e.g., "The general UM COVID-19 prevention guidelines are difficult to adhere to") were measured. All items were measured on a 7-point Likert scale (see Supplementary File 1 for the full questionnaire).

\section{Keeping $1.5 m$ distance}

Keeping 1.5m distance intention was measured with three items: "For the next two months I intend to: (a) keep $1.5 \mathrm{~m}$ distance from the people close to me, (b) keep $1.5 \mathrm{~m}$ 
distance from all other people, and (c) avoid crowds. Additionally, attitude (2 items), perceived norm (2 items), self-efficacy ( 2 items), risk perception ( 1 item), risk perception belief (1 item), attitudinal beliefs ( 6 items), perceived norm belief ( 1 item), self-efficacy beliefs ( 7 items) and habits (a 6-item scale, $\Omega=.83$; e.g., "Coming closer than $1.5 m$ to other people is something that ... I may do without thinking”; for the use of $\Omega$, see Crutzen \& Peters, 2017). We provided $\Omega$ scores where necessary. If we did not indicate $\Omega$, that means that each item was separately assessed in the CIBER analysis since each relevant item is likely to be a target for an intervention. All items used similar formulations as the items as described above (see Adherence to COVID-19-guidelines of the university) and were measured on a 7-point Likert scale (see Supplementary File 1 for the exact questions).

\section{Testing and isolating}

To measure testing and isolating behavior, one measure of intention was included (i.e., "For the next two months, I intend to ... get tested and stay at home as a precaution until I have the test results if I have cold-related symptoms or a high temperature (fever)"). Additionally, attitude (2 items), perceived norm (2 items), self-efficacy ( 2 items), risk perception ( 1 item), attitudinal beliefs ( 6 items), and self-efficacy beliefs ( 2 items) were measured. All items were again similar to the items described above and measured on a 7point Likert scale (see Supplementary File 1 for the exact questions).

\section{Data analyses}

IBM SPSS Statistics 26 was used for descriptive analyses, e.g., frequencies, means $(M)$ and standard deviations $(S D)$. Correlations between students' adherence behaviors to guidelines and their intentions to adhere to guidelines, and correlations between intentions and their determinants were analyzed. In our analysis, we did not control for age or gender. Intervening in the university setting did not allow for targeting specific students based on their gender and 
age. The 'constraints' of the intervention setting consisted of using communication channels within the university and changes in the environment (e.g., providing certain facilities) that affected all students (regardless of their gender and age).

To select the most relevant determinants, i.e., to what extent the determinants were correlated with intention (as most important predictor of behavior), and to what extent there was room for improvement, we used the CIBER approach, which visualizes the data and illustrates the univariate distribution of each item in one panel and the association between behavior/determinant and determinants in another panel (see Figure 1; Crutzen et al., 2017; Peters \& Crutzen, 2018). It is necessary to combine these two types of analyses when establishing relevance. Assessing the associations of determinants with behavior and/or determinants is important because those determinants that are not associated with behavior and/or more proximal determinants will often be the least likely candidates to intervene upon. The univariate distributions are also important because bimodal distributions may be indicative of subgroups, and strongly skewed distributions have implications for how a determinant should be targeted. For example, if a determinant is positively associated with behavior but left-skewed, most population members already have the desired value (for positively formulated questions), so it should merely be reinforced in an intervention.

Conversely, right-skewed positively associated determinants imply a need for change, as most population members do not have the desired value yet. This latter category of determinants would be more viable intervention targets as there is more room for improvement. To create the CIBER plots, we used the 'behaviorchange' R package (Peters, 2021).

\section{Results}

A total of 907 students (all UM-student-panel members) were invited to participate, with the prerequisite of having the intention to visit the university in the next two months; 328 students (36.2\%) responded to the survey (after removing 57 responses: poor response quality (e.g., 
consistency of answers, straightlining and completion time) [n=2], drop-out/incomplete questionnaire $[\mathrm{n}=55])$. Among those 328 students, 69 stated they do not intend to visit the university in the next two months and 4 students mentioned they did not visit the university in the past two months, and thereby did not have behavioral data. Hence, 255 students $(75.7 \%$ female) were included in the data analyses. The mean age of students was 21.0 years $(S D=$ 2.7) and 50 students (19.6\%) indicated that they are living alone. More detailed background characteristics of the sample are provided in Table 1.

\begin{tabular}{|c|c|c|c|}
\hline Students & $\mathrm{N}(\%)$ & & \\
\hline Female & $193(75.7 \%)$ & & \\
\hline Age in years $(M+S D)$ & $21.0(2.7)$ & & \\
\hline Study year & & Faculty* & $\mathrm{N}(\%)$ \\
\hline Bachelor year 1 & $81(31.8 \%)$ & FHML & $91(35.7 \%)$ \\
\hline Bachelor year 2 & $52(20.4 \%)$ & FASoS & $28(11 \%)$ \\
\hline Bachelor year 3 & $48(18.8 \%)$ & FPN & $29(11.4 \%)$ \\
\hline Pre-master & $2(0.8 \%)$ & SBE & $39(15.3 \%)$ \\
\hline Master year 1 & $51(20 \%)$ & $\mathrm{FdR}$ & $18(7.1 \%)$ \\
\hline Master year 2 & $17(6.7 \%)$ & FSE & $50(19.6 \%)$ \\
\hline Master year 3 & $4(1.6 \%)$ & & \\
\hline \multicolumn{2}{|l|}{ Living situation } & \multicolumn{2}{|c|}{ *FHML: Faculty of Health, Medicine and Life } \\
\hline I live alone & $50(19.6 \%)$ & \multicolumn{2}{|c|}{ Sciences; FASoS: Faculty of Arts and Social Sciences } \\
\hline I live with my parent(s)/caretaker(s) & $51(20 \%)$ & \multicolumn{2}{|c|}{ FPN: Faculty of Psychology and Neuroscience; SBE: } \\
\hline I live with my partner & $20(7.8 \%)$ & \multicolumn{2}{|c|}{ School of Business and Economics; FdR: Faculty of } \\
\hline I live with people other than the abovementioned & $134(52.5 \%)$ & Law; FSE: Faculty of Sc & eering. \\
\hline
\end{tabular}

Table 1. Background Characteristics of the Sample $(N=255)$ 


\section{Adherence to COVID-19-guidelines of the university}

Students showed high adherence to COVID-19 university guidelines based on selfreported measures. In the past two months, $34.1 \%$ (almost) always kept $1.5 \mathrm{~m}$ distance from other people $(M=5.76 ; S D=1.19 ; M d n(I Q R)=6.00(5.00$ to 7.00$)) ; 24.7 \%$ (almost) always avoided situations where one cannot keep $1.5 \mathrm{~m}$ distance $(M=5.29 ; S D=1.49 ; \operatorname{Mdn}(I Q R)=$ 5.00 (4.00 to 6.00$)), 51.4 \%$ (almost) always avoided crowds $(M=6.13 ; S D=1.12 ; M d n(I Q R)$ $=7.00$ (5.00 to 7.00)), $75.3 \%$ (almost) always disinfected their hands upon entering the university buildings $(M=6.46 ; S D=1.14 ; M d n(I Q R)=7.00(7.00$ to 7.00$)), 87.1 \%$ stated that they (almost) always refrained from shaking hands in the past two months $(M=6.76 ; S D$ $=.70 ; \operatorname{Mdn}(I Q R)=7.00(7.00$ to 7.00$))$. A total of $77.6 \%$ students did not have cold-related symptoms or a high temperature/fever in the past two months. Of the remaining $22.4 \%$ students who had symptoms or fever, $77.2 \%$ stated that they got tested for COVID-19, and $90.9 \%$ of students who got tested stated that they (almost) always stayed at home as a precaution until they had the test results. The mean score of students' intention to adhere to guidelines was $M=6.33(S D=.75) ; M d n(I Q R)=6.50(6.00$ to 6.83). Moreover, students' intentions to adhere to COVID-19-guidelines of the university was positively correlated with their adherence behaviors ( $r$ 's ranging from $.36-.68$ for the different behaviors; all $p$ 's $<$ .001 ; note that getting tested and isolating behavior was not included here).

\section{Selecting the most relevant determinants and underlying beliefs}

In this section, we will report the results for 'Adherence to COVID-19-guidelines of the university' in detail, to illustrate the systematic approach for selecting determinants and their underlying beliefs, based on the CIBER plots (Peters \& Crutzen, 2018). For keeping distance and getting tested/isolating, we will report the detailed analyses in the

Supplementary Materials File 4, and just report summaries of the selected determinants and beliefs in this text. 
Attitude: Both attitude questions (bad - good - further referred to as attitude/good; and unpleasant - pleasant - further referred to as attitude/pleasant) were positively associated with intention to adhere to guidelines ( $r=.50$ and $r=.31$ respectively; both $p$ 's $<.001)$. However, attitude/good had a very high mean score $(M=6.60 ; S D=.85 ; \operatorname{Mdn}(\operatorname{IQR})=7.00$ (6.00 to 7.00)) as opposed to attitude/pleasant $(M=4.20 ; S D=1.67 ; M d n(I Q R)=4.00(3.00$ to 5.00)). Although both attitude items were positively correlated with intention, for attitude/good (see Figure 1, right panel), students were already convinced that adhering to COVID-19-guidelines of the university would be good (see Figure 1, left panel). Therefore, there is less to no room for improvement for attitude/good, while attitude/pleasant could be targeted with accepting the disadvantages in balance with the evident advantages.

Perceived norm: Both perceived norm items (i.e., "Most people like me always adhere to the general UM COVID-19 prevention guidelines" further referred to as PN/like me; and "Most people who are important to me think I should adhere to the general UM COVID-19 prevention guidelines" further referred to as PN/important others) were positively correlated with intention ( $r=.35$ and $r=.55$ respectively; both $p$ 's $<.001)$. When analyzing the mean scores of both items separately, perceived norm/important others and perceived norm/like me had mean scores of $M=6.14(S D=1.05) ; M d n(I Q R)=6.00(6.00$ to 7.00$)$ and $M=5.27(S D=1.37) ; M d n(I Q R)=5.00(5.00$ to 6.00$)$ respectively. This indicates that PN/like me had a high relevance and more room for improvement than PN/important others, although both items are relevant targets for an intervention (see Figure 1). Both could become more positive with an appropriate intervention.

Self-efficacy: Both self-efficacy items ("I am confident that if I want to, I can adhere to the general UM COVID-19 prevention guidelines" and "Always adhering to the general UM COVID-19 prevention guidelines is up to me", further referred to as SE/confident and $\mathrm{SE} / \mathrm{up}$ to me) were positively correlated with intention $(r=.42, p<.001$, and $r=.19 ; p<.01$ 
respectively). Both had relatively high mean scores (SE/confident: $M=6.38, S D=.94, M d n$ $(I Q R)=7.00$ (6.00 to 7.00); SE/up to me: $M=5.42, S D=1.68, M d n(I Q R)=6.00$ (4.00 to 7.00)), which state that students were already confident that they can adhere to the guidelines, meaning there is less room for improvement. Even though students' individual scores for SE/up to me were more scattered over the scale, the relevance was relatively low because it was only weakly correlated with intention (see Figure 1).

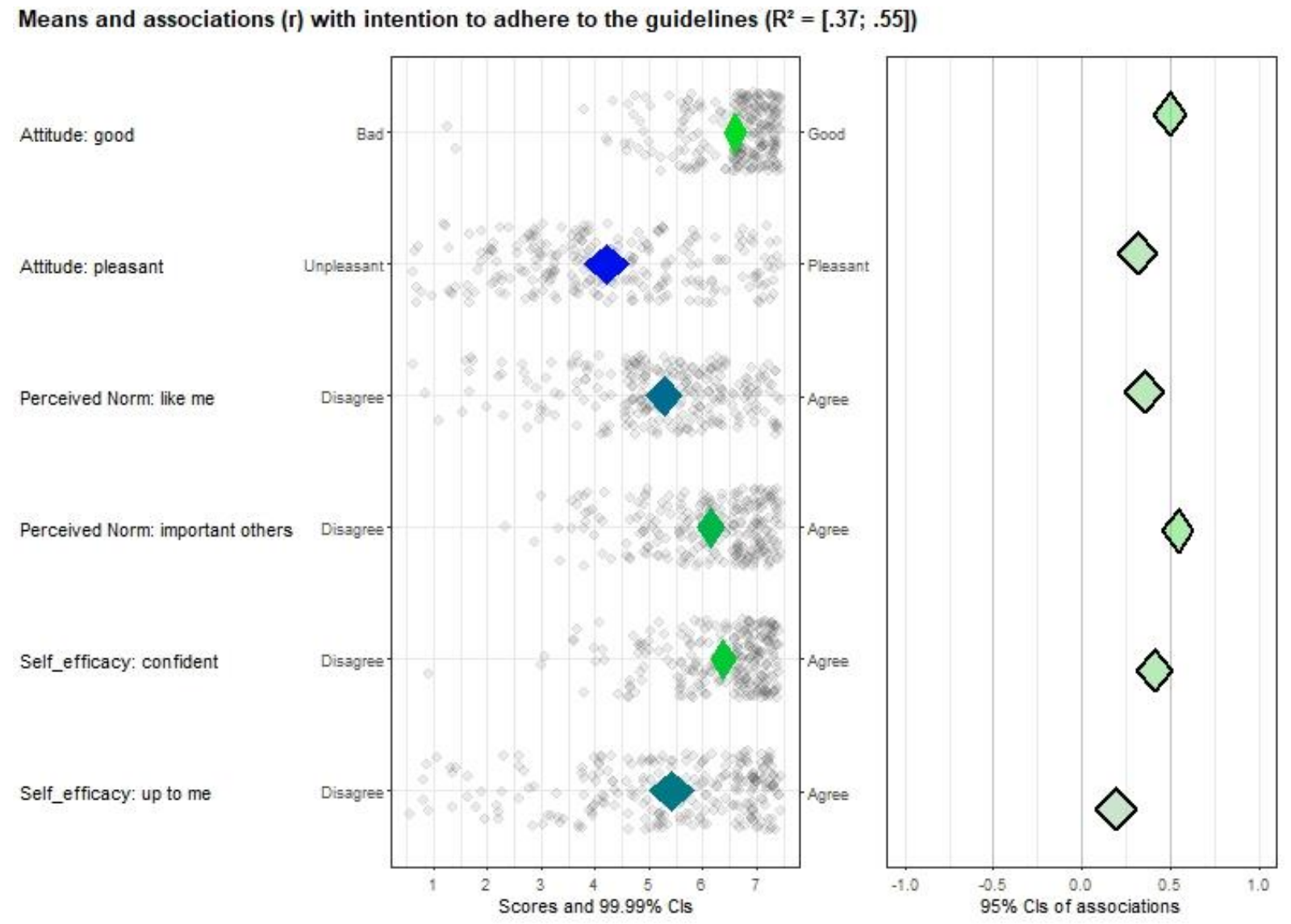

Note: On the left, the names of the determinants are displayed (or question items in Figure 2). The left-hand panel includes mean scores of determinants with $99.99 \%$ confidence intervals. The colors of the diamonds demonstrate the skewness of the distribution: red is positively-skewed and green is negatively-skewed. The righthand panel shows the association between the target variable (intention) and the determinants (e.g., attitude and perceived norm).

Figure 1. Univariate distributions of determinants and their association with intention to adhere to the guidelines. 
Risk perception beliefs: Out of four risk perception beliefs, 'I am not afraid of contracting COVID-19 because I am young. 'was negatively correlated with intention ( $r=$ $.33 ; p<.001$; see Figure 2, right panel) and the mean score was close to the middle of the scale $(M=3.05 ; S D=2.06 ; M d n(I Q R)=3.00$ (1.00 to 5.00); see Figure 2, left panel). This suggests that this particular risk perception belief is highly relevant as a target for future interventions, stressing that being young is not a guarantee for avoiding serious negative consequences of contracting COVID-19. All other risk perception beliefs were not significantly correlated with intention (all $r$ 's ranged between -.12 and .10 , with $p$ 's $>.05$ ).

Attitudinal beliefs: Attitudinal beliefs "The general university COVID-19 prevention guidelines are irrelevant for our generation" and "The general university COVID-19 prevention guidelines cause me to become tired of the whole situation" both had negative correlations with intention to adhere to guidelines $(r=-.37 ; p<.001$ and $r=-.22 ; p<.01)$. In addition, "The general university COVID-19 prevention guidelines are well-organized" and "I feel responsible for telling people around me to adhere to the guidelines" was positively correlated with intention ( $r=.32$ and $r=.37$ respectively, $p$ 's $<.001)$. Other attitudinal beliefs had no association with intention. As students showed strong disagreement with the item "The general university COVID-19 prevention guidelines are irrelevant for our generation", and because they already agreed that the guidelines are well-organized, these two items had relatively low potential for change. On the other hand, as the individual scores were all over the scale and the mean score was in the middle of the scale, the items of 'I feel responsible for telling people around me to adhere to the guidelines' and 'The general university COVID-19 prevention guidelines cause me to become tired of the whole situation' were highly relevant.

Perceived norm belief: The belief "My teachers/tutors at the university care about the general UM COVID-19 prevention guidelines" was positively correlated with students' intention to adhere to the guidelines $(r=.35 ; p<.001)$. Since students are already convinced 
about this $(M=5.74 ; S D=1.27 ; M d n(I Q R)=6.00(5.00$ to 7.00$))$, it had a relatively low relevance as a target for change.

Self-efficacy beliefs: Both self-efficacy beliefs ("The general university COVID-19 prevention guidelines are difficult to adhere to" and 'The information we receive about the general UM COVID-19 prevention guidelines is confusing') had a negative correlation with intention (respectively: $r=-.30 ; M=2.80 ; S D=1.52 ; M d n(I Q R)=3.00(2.00$ to 4.00$) ; r=-$ $.21 ; M=2.78 ; S D=1.55 ; M d n(I Q R)=2.00$ (2.00 to 4.00); see Figure 2). Both beliefs could become more positive (meaning lower scores) through an intervention, especially the first, increasing students' self-efficacy to adhere to the guidelines.

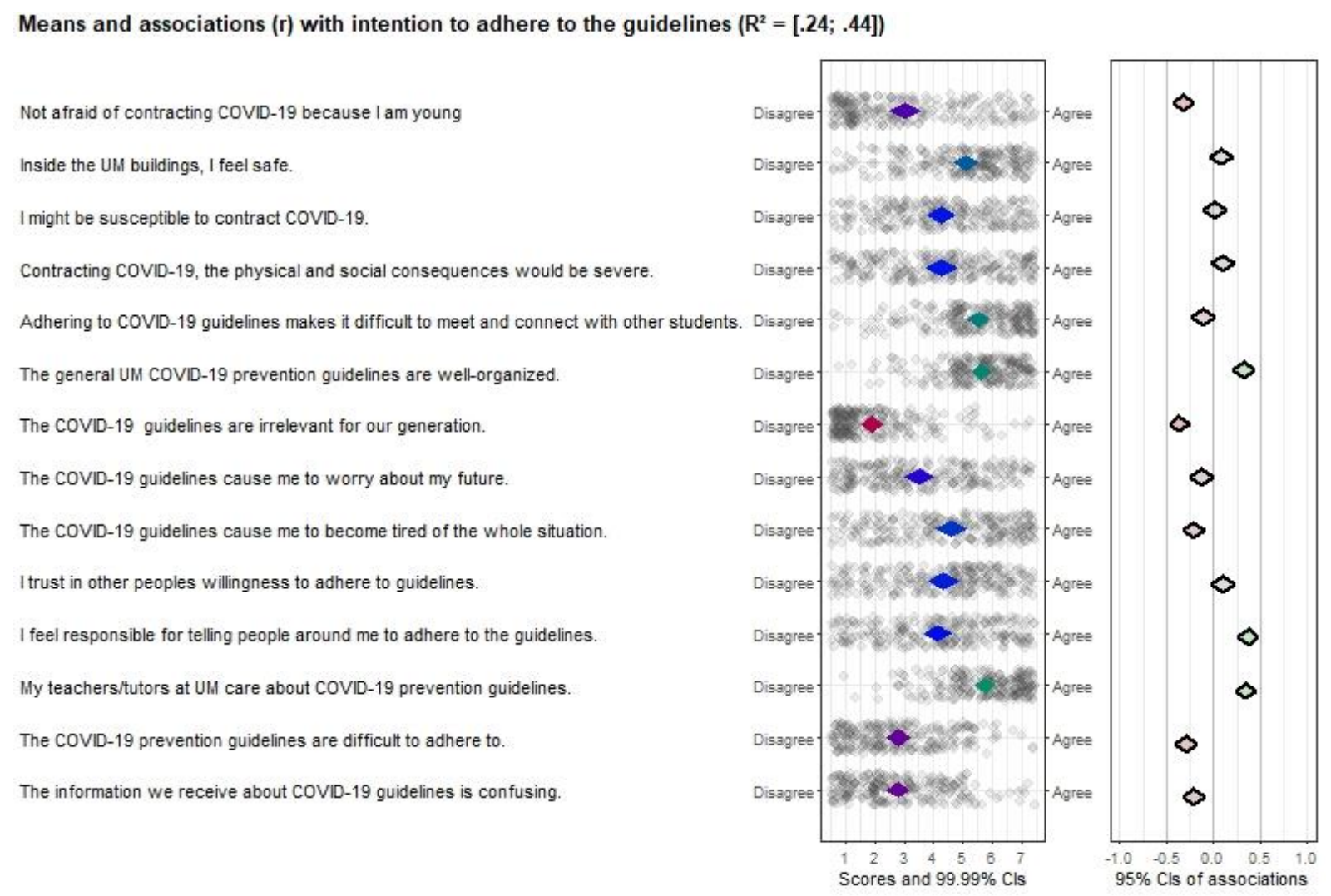

Figure 2. Univariate distributions of underlying beliefs and their association with intention to adhere to the guidelines 


\section{Summary of keeping $1.5 \mathrm{~m}$ distance}

Keeping distance was subdivided into three categories: keeping distance from people close to me, keeping distance from all other people, and avoiding crowds. In this section only the most relevant determinants and underlying beliefs are mentioned. Although relevance is subjective, we define most relevant as: 1) the correlation with intention is at least moderate $(r>.30)$ AND 2) the mean score on a variable could potentially improve with at least 1 point (on the 7-point Likert-scale). Note that for each intervention a different focus can be decided, and with changing this definition, a ranking of most relevant determinants and underlying beliefs can be established. The above-mentioned rule to select the most relevant determinants/beliefs was also used for getting tested/isolating. All CIBER plots can be found in Supplementary materials File 3.

For the intention to keep distance to people close to the individual and to keep distance from all other people, both perceived norm (important others) and self-efficacy (confident) were seen as relevant and changeable. For keeping distance to all other people (contrary to people close to an individual), also one's risk perception $(r=.40 ; M=5.69 ; S D=$ 1.33; $\operatorname{Mdn}(I Q R)=6.00(5.00$ to 7.00$))$, habit $(r=-.31 ; M=4.26 ; S D=1.27 ; M d n(I Q R)=$ 4.33 (3.50 to 5.17)), and attitudinal belief "Keeping 1.5 m distance would ensure that other people do not contract COVID-19 through me" $(r=.31 . ; M=5.76 ; S D=1.29 ; \operatorname{Mdn}(I Q R)=$ $6.00(5.00$ to 7.00$))$ can be seen as important targets for intervention development. No additional determinants or underlying beliefs met our criteria of being relevant when it comes to "Avoiding crowds".

\section{Summary of results on getting tested/isolating}

Although many determinants related to getting tested and isolating when having COVID-19 related complaints had moderate to high correlations with intention $(N=255)$, 
only one attitudinal belief met our criteria for being highly relevant: "Testing and isolating means taking care of yourself' $(r=.46 ; \%=5.96 ; S D=1.35 M d n(I Q R)=6.00$ (5.00 to 7.00)). However, in hindsight this statement might also have been interpreted as "only yourself", which makes it ambiguous.

\section{Discussion}

The aim of the study was to identify the most relevant determinants of students' adherence to general COVID-19-guidelines of the university. Students mostly adhere to guidelines, but there is room for improvement. In our study, we gave some insights in how to select relevant and changeable determinants of adherence for future intervention development.

Similar to our earlier and the current study, Blake et al. (2021) found that students mostly adhere to guidelines. However, in some situations (e.g., due to the environment), they experience difficulties. Barrett and Cheung (2021) and Wismans et al. (2020) reported that college students perceived several barriers for successful adherence to preventive measures, in particular in relation to social distancing. In our earlier qualitative study (Varol et al., 2021), we also found that students were willing to adhere to guidelines within the university buildings but besides mentioning several facilitators (e.g., the infrastructure of the buildings and reminders from staff) they perceived barriers for adherence to the behavioral guidelines, for example difficulties with telling friends to follow the regulations. Also, some students stated that they are not afraid of COVID-19 because they are young, which makes it difficult for them to see the need for following the guidelines.

Reicher and Drury (2021) claim that the main problem is not people's lack of willingness to adhere to guidelines. Our studies also point out that students mostly intend to adhere to COVID-19 guidelines, however, they might require the help of intervention developers to enhance the adherence. In order to change a behavior, we first need to know 
what to target (i.e., determinants (and underlying beliefs) of students' behaviors). Therefore, in the current study, we selected the determinants (and underlying beliefs) that have room for improvement, which makes them clear targets for an intervention, which we will describe in the next section (see Translating our findings into a small intervention).

This study had several limitations. First, due to the nature of our survey, findings are based on self-report. Therefore, the actual behavior and self-reported behavior might be different. However, in our earlier qualitative study, based on their observations, stewards/security people reported that students adhere to guidelines and are willing to do so. Therefore, high adherence rates found in the current study might not be due to social desirability but what we observe happening in practice within our university setting. Second, only students who are willing to visit the university in the next two months were invited and we do not know if the determinants of students who are willing to visit the university in the next two months and other students are the same. Third, although it was explicitly mentioned at the beginning of the questionnaire that we were not looking for desirable answers, social desirability might still be a factor that affected the results (however also see limitation 1). Fourth, we could not assess the determinants of all behaviors that were included in the guidelines of the university as a longer questionnaire might increase the drop-out rate/uncompleted responses. Fifth, the conditions constantly change, therefore, the guidelines and measures also. Currently (April 2021), all universities have moved to mainly onlinelearning. The determinants might be different when vaccination rates have reached a critical threshold. Of course, that situation was not at hand during data collection for the current study, so the study might be repeated later to see how determinants change comparing a prevaccination vs. post-vaccination situation. However, our findings are still helpful for universities to create safe environments for their students when the universities are reopening. Moreover, one of the goals of this paper is to demonstrate how the findings of a determinant 
study can be translated into an intervention, so that others can use a similar approach. Sixth, our focus is not on theory or generalizable data; our focus is on the process of developing an intervention for the specific situation at our university at that time and that process is hopefully generalizable to other settings and times. Lastly, we mainly utilized the theories of Reasoned Action Approach and Protection Motivation Theory and the results of our qualitative study in the selection of determinants and formulation of questions related to these determinants. Hence, there might be other determinants that affect students' adherence behaviors that are not included in the study at hand. However, building on the results of the qualitative research among this target group, we are convinced that we covered the most salient beliefs.

\section{Translating our findings into a small intervention}

Based on the findings of this study, the most relevant determinants and underlying beliefs behind students' adherence to the guidelines were selected. While the Christmas/New Year break was approaching, a small intervention, a New Year's message to students, was created which included the intervention messages targeting determinants of students' adherence to guidelines, and specific behaviors, in this case specifically keeping distance from others during the Christmas break, as most students go visiting their families and friends; see Figure 3. Embedded in the New Year's message was a short documentary (Marketing \& Communications Maastricht University Office, 2020), in which students who have had COVID-19 share what this disease has done and is still doing to them. They also let us know what they think of the measures. This video was developed independently, but it provided an excellent 'real life' input to the message and both complement each other.

The New Year's message was carefully crafted combining the outcomes of our determinants' study and the available literature on effective communication for behavior change. Based on the observed risk behaviors from the survey, and their determinants, the 
main focus was on (1) the limited group of students who indicated that they are "not afraid of contracting COVID-19 because I am young"; (2) students who feel responsible for telling other people "to adhere to the guidelines"; (3) students who "become tired of the whole situation"; and (4) students who indicate that the "prevention guidelines are difficult to adhere to". These four determinants are targeted by appropriate behavior-change methods, derived from Intervention Mapping (Bartholomew-Eldredge et al., 2016: chapter 6; Kok et al., 2016: supplementary file). In these references, those methods, and their so-called parameters for effectiveness, are systematically described. See Figure 3 for the New Year's message, with the theoretical methods indicated in the text and described below the text. In the message, feedback and reinforcement are two examples of methods that were used. Feedback was used in the following intervention message: "Many of you have taken responsibility, for yourselves and for others. You have consistently followed the government guidelines both on and off the UM campus." In terms of determinants, this message does not deny the difficulty in adhering to guidelines but it does show that most students (as indicated in our survey) do adhere to this. This is also aimed to be reinforced in the next intervention message: "That makes us proud and grateful". For those students that have difficulty adhering to guidelines, amongst others, the method of planning coping responses was used. This consisted of providing tips on how to deal with high-risk situations in the upcoming Christmas/New Year break. When looking at the parameters for effectiveness for planning coping responses, there are two aspects: (1) identification of high-risk situations and (2) practice of coping response. This shows that adequate translation of the method into practical applications (in this case a New Year's message) is constrained by the vehicle used to deliver the intervention messages. In this general New Year's message, it was possible to identify high-risk situations and communicate those to students in combination with a number of practical tips on what to do. However, actual practicing of coping responses was left to the responsibility of students. 


\section{Good preparation for a better 2021}

For most of us, 2020 has not been easy. People became seriously ill from COVID-19. Some lost loved ones to the virus. And our social lives were largely put on hold ${ }^{a}$. Many of you have taken responsibility, for yourselves and for others. You have consistently followed the government guidelines both on and off the UM campus ${ }^{b}$. That makes us proud and gratefulc.

Unfortunately, the end of the pandemic is not yet in sight ${ }^{\mathrm{a}}$. That is why we are looking ahead to the upcoming holiday period and the beginning of 2021. Our message: please stay the course so that 2021 will be a better year for all of $u s^{c}$.

\section{Even if you are young!}

It is a well-established fact that young people can transmit COVID-19 without experiencing any symptoms themselves ${ }^{d}$. So, even if you are young and think you are protected against the effects of COVID-19, you can still be a danger to others ${ }^{d}$. Imagine how you would feel if someone else who is vulnerable were to become infected with COVID-19 because of you ${ }^{e}$. This could happen while you are visiting your family during the Christmas holidays, as well as if you stay in Maastricht and fail to follow the safety protocols ${ }^{f}$. Therefore, however healthy and strong you may feel, make sure you keep your distance and follow the guidelines as long as the pandemic persists ${ }^{g}$. This is how you can help to ensure that society returns to normal ${ }^{\mathrm{h}}$.

Sometimes young people do not feel sick if they have COVID-19. But often they do'. In this short documentary, students who have had COVID-19 share what this disease has done and is still doing to them ${ }^{\mathrm{j}, \mathrm{k}}$. They also let us know what they think of the measures ${ }^{1, m}$.

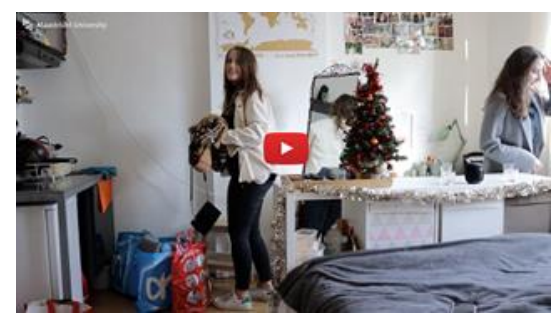

\section{Good preparation...}

Nobody underestimates how difficult it is - keeping your distance, few social contacts, not celebrating the holidays with your entire family and all your friends ${ }^{a}$. You can make it easier on yourself by thinking about it now. Prepare in advance, so you know what you are going to do to stay healthy and safe during the holiday period ${ }^{n}$. That way, you know what lies ahead. For example, you could follow these tips:

- Discuss in advance with your family and friends how to get through the holidays safely. For instance, create a top-10 list of safe activities that you can do together and make agreements on how to protect each other ${ }^{n}$.

- Head outdoors and take a walk in nature while staying 1.5 meters away from each other ${ }^{n}$.

- Keeping a physical distance doesn't mean you can't have social contacts. Celebrate New Year's via Zoom or another platform ${ }^{\mathrm{n}}$. If we all do it, this will hopefully be the first and last time it needs to be done ${ }^{\circ}$. 
- Organize social gatherings in such a way that it is easy to keep your distance ${ }^{n}$.

- If you have friends who don't want to follow the guidelines, don't invite them ${ }^{p}$. Also, don't visit people who are breaking the rules ${ }^{p}$. That might sound strict, but by doing this, you are helping a huge group of people ${ }^{c}$. Remember, the more people and the closer together they are, the faster the virus spreads ${ }^{\mathrm{a}, \mathrm{d}}$.

- Very important: don't go to a social gathering if you have symptoms of COVID-19a,d. You can always call for a quick and easy test and stay at home until you have the results ${ }^{n}$. Of course, don't visit anyone if you have any symptoms, even if they are mildn.

...makes for a better 2021!

A vaccine will be available in 2021. It will take a while before everyone gets their turn, but then we can move on to a new normalc. Until then, as a UM community, we will also follow the guidelines. It is a question of perseverance, however difficult it may be, but we are doing it for our friends, our families, the vulnerable in our society, and for ourselves ${ }^{c}$. Together, we will overcome ${ }^{\mathrm{h}, \mathrm{l}}$.

Happy holidays and come back healthy!

\begin{tabular}{|c|c|c|}
\hline a: Consciousness raising & g: Goal setting & m: Information about others' approval \\
\hline b: Feedback & h: Environmental re-evaluation & $\mathrm{n}$ : Planning coping responses \\
\hline c: Reinforcement & i: Personalize risk & o: Environmental re-evaluation \\
\hline d: Scenario-based risk information & j: Modeling & $\mathrm{p}$ : Resistance to social pressure \\
\hline e: Anticipated regret & k: Cultural similarity & \\
\hline f: Punishment & I: Mobilizing social support & \\
\hline
\end{tabular}

All these methods and their parameters are described in: Bartholomew-Eldredge et al., 2016: chapter 6, and: Kok et al., 2016: supplementary file (open access)

Figure 3. New Year's message for the students, plus identification of underlying behavior change methods

\section{Conclusion}

This study identifies the relevant determinants and underlying beliefs of students' adherence to COVID-19-guidelines. Moreover, it is a showcase demonstrating how results of a determinant study can be used when developing intervention messages. We do not know for sure if this intervention had the desired effect, but we are convinced that we have optimized 
the likelihood of achieving the desired effect by following the optimal theory- and evidencebased process in a short time period. That process can be repeated in comparable needs and times, even in different settings, where resources (time, budget) are constrained.

\section{Disclosure of interest}

The authors report no conflict of interest. 


\section{References}

Adhikari, S. P., Meng, S., Wu, Y. J., Mao, Y. P., Ye, R. X., Wang, Q. Z., ... \& Zhou, H. (2020). Epidemiology, causes, clinical manifestation and diagnosis, prevention and control of coronavirus disease (COVID-19) during the early outbreak period: a scoping review. Infectious Diseases of Poverty, 9(1), 1-12. https://doi.org/10.1186/s40249-020-00646-X

Ajzen, I. (2011). The theory of planned behavior: Reactions and reflections. Psychology \& Health, 26(9), 1113-1127. https://doi.org/10.1080/08870446.2011.613995

Auger, K. A., Shah, S. S., Richardson, T., Hartley, D., Hall, M., Warniment, A., ... \& Thomson, J. E. (2020). Association between statewide school closure and COVID-19 incidence and mortality in the US. JAMA, 324(9), 859-870.

https://doi.org/10.1001/jama.2020.14348

Barrett, C., \& Cheung, K. L. (2021). Knowledge, socio-cognitive perceptions and the practice of hand hygiene and social distancing during the COVID-19 pandemic: a crosssectional study of UK university students. BMC public health, 21(1), 1-18. https://doi.org/10.1186/s12889-021-10461-0

Bartholomew-Eldredge, L. K., Markham, C. M., Ruiter, R. A., Fernández, M. E., Kok, G., \& Parcel, G. S. (2016). Planning health promotion programs: an Intervention Mapping approach. John Wiley \& Sons.

Blake, H., Knight, H., Jia, R., Corner, J., Morling, J. R., Denning, C., ... \& Vedhara, K. (2021). Students' views towards SARS-CoV-2 mass asymptomatic testing, social distancing and self-Isolation in a university setting during the COVID-19 pandemic: A qualitative study. https://doi.org/10.20944/preprints202101.0399.v1 
Crutzen, R., \& Peters, G. J. Y. (2017). Scale quality: alpha is an inadequate estimate and factor-analytic evidence is needed first of all. Health Psychology Review, 11(3), 242247. https://doi.org/10.1080/17437199.2015.1124240

Crutzen, R., Peters, G. J. Y., \& Noijen, J. (2017). Using confidence interval-based estimation of relevance to select social-cognitive determinants for behavior change interventions. Frontiers in Public Health, 5, 165. https://doi.org/10.3389/fpubh.2017.00165

Fauci, A. S., Lane, H. C., \& Redfield, R. R. (2020). Covid-19-Navigating the Uncharted. https://doi.org/10.1056/NEJMe2002387

Fernandez, M. E., Ruiter, R. A. C., Markham, C. M., \& Kok, G. (2019). Theory-and evidence-based health promotion program planning: Intervention Mapping. Frontiers in Public Health, 7, 209. https://doi.org/10.3389/fpubh.2019.00209

Fishbein, M., \& Ajzen, I. (2010). Predicting and changing behavior: The reasoned action approach. New York: Psychology Press

Flycatcher Internet Research. (2021). About us. https://www.flycatcher.eu/en/Home/OverOns

Head, J. R., Andrejko, K., Cheng, Q., Collender, P. A., Phillips, S., Boser, A., ... \& Remais, J. V. (2020). The effect of school closures and reopening strategies on COVID-19 infection dynamics in the San Francisco Bay Area: a cross-sectional survey and modeling analysis. medRxiv. https://doi.org/10.1101/2020.08.06.20169797

Kok, G. (2014). A practical guide to effective behavior change: How to apply theory- and evidence-based behavior change methods in an intervention. European Health Psychologist, 16(5), 156-170. https://doi.org/10.31234/osf.io/r78wh 
Kok, G., Gottlieb, N. H., Peters, G. J. Y., Mullen, P. D., Parcel, G. S., Ruiter, R. A. C., ... \& Bartholomew, L. K. (2016). A taxonomy of behaviour change methods: an Intervention Mapping approach. Health Psychology Review, 10(3), 297-312.

Maddux, J. E., \& Rogers, R. W. (1983). Protection motivation and self-efficacy: A revised theory of fear appeals and attitude change. Journal of Experimental Social Psychology, 19(5), 469-479. https://doi.org/10.1016/0022-1031(83)90023-9

Marketing \& Communications Maastricht University Office (Ron van der Bolt, film; Annabel Reke, producer). 2020. Three UM students who have had COVID-19 share how it affected them [Streaming video]. Available from https://maastrichtuniversity.bbvms.com/view/default_videoteam/4062759.html

Peters, G.-J. Y. (2014). A practical guide to effective behavior change: how to identify what to change in the first place. European Health Psychologist, 16(4), 142-155. https://doi.org/10.31234/osf.io/hy $7 \mathrm{mj}$

Peters G.-J. Y. (2021). behaviorchange: Tools for behavior change researchers and professionals. R package version 0.3 https://r-packages.gitlab.io/behaviorchange

Peters, G. J. Y., Abraham, C., \& Crutzen, R. (2012). Full disclosure: doing behavioural science necessitates sharing. The European Health Psychologist, 14(4), 77-84.

Peters, G.-J. Y. \& Crutzen, R. (2018). Establishing determinant importance using CIBER: an introduction and tutorial. The European Health Psychologist, 20(3), pp. 484-494. https://doi.og/10.31234/osf.io/5wjy4

Petretto, D. R., Masala, I., \& Masala, C. (2020). School Closure and Children in the Outbreak of COVID-19. Clinical Practice and Epidemiology in Mental Health, 16, 189. https://dx.doi.org/10.2174\%2F1745017902016010189 
Reicher, S., \& Drury, J. (2021). Pandemic fatigue? How adherence to covid-19 regulations has been misrepresented and why it matters. BMJ, 372 . https://doi.org/10.1136/bmj.n137

Rothan, H. A., \& Byrareddy, S. N. (2020). The epidemiology and pathogenesis of coronavirus disease (COVID-19) outbreak. Journal of Autoimmunity, 102433. https://doi.org/10.1016/j.jaut.2020.102433

Shereen, M. A., Khan, S., Kazmi, A., Bashir, N., \& Siddique, R. (2020). COVID-19 infection: Origin, transmission, and characteristics of human coronaviruses. Journal of Advanced Research. https://doi.org/10.1016/j.jare.2020.03.005

Van Bavel, J. J., Baicker, K., Boggio, P. S., Capraro, V., Cichocka, A., Cikara, M., ... \& Drury, J. (2020). Using social and behavioural science to support COVID-19 pandemic response. Nature Human Behaviour, 4(5), 1-12. https://doi.org/10.1038/s41562-020-0884-Z

Varol, T., Schneider, F., Mesters, I., Crutzen, R., Ruiter, R. A. C., Kok, G., \& ten Hoor, G. (2021). University Students' Adherence to the COVID-19-guidelines: A Qualitative Study on Facilitators and Barriers. Health Psychology Bulletin, 5(1), 114-123. http://doi.org/10.5334/hpb.32

Von Elm, E., Altman, D. G., Egger, M., Pocock, S. J., Gøtzsche, P. C., \& Vandenbroucke, J. P. (2007). The Strengthening the Reporting of Observational Studies in Epidemiology (STROBE) Statement: Guidelines for Reporting Observational Studies. Annals of Internal Medicine, 147(8), 573-577. https://doi.org/10.7326/0003-4819-147-8-200710160-00010

Wismans, A., Letina, S., Thurik, R., Wennberg, K., Franken, I., Baptista, R., ... \& Torrès, O. (2020). Hygiene and social distancing as distinct public health related behaviours 
among university students during the COVID-19 Pandemic. Social Psychological Bulletin, 15(4), 1-26. https://doi.org/10.32872/spb.4383

Wood, W., \& Rünger, D. (2016). Psychology of habit. Annual Review of Psychology, 67. https://doi.org/10.1146/annurev-psych-122414-033417

World Health Organization. (2020). Behavioural considerations for acceptance and uptake of COVID-19 vaccines: WHO technical advisory group on behavioural insights and sciences for health, meeting report, 15 October 2020. (accessed January 2021) https://apps.who.int/iris/bitstream/handle/10665/337335/9789240016927-eng.pdf

Ziauddeen, N., Woods-Townsend, K., Saxena, S., Gilbert, R., \& Alwan, N. A. (2020). Schools and COVID-19: Reopening Pandora's Box? Public Health in Practice, 1, 100039. https://doi.org/10.1016/j.puhip.2020.100039 